\title{
Analysis of Topographic Decorrelation in SAR Interferometry Using Ratio Coherence Imagery
}

\author{
Hoonyol Lee, Student Member, IEEE, and Jian Guo Liu
}

\begin{abstract}
Topographic decorrelation due to the local surface slope has been an obstacle to interferometric synthetic aperture radar (InSAR) applications. A modified spatial decorrelation function is derived as a function of the baseline and topography. This function explains the origin of the total topographic decorrelation phenomenon on the slopes directly facing radar illumination and layover, which may mislead InSAR coherence image interpretation. We define critical terrain slope (or critical incidence angle) as the angle for which two SAR signals completely decorrelate regardless of surface stability. It is found that the width of the critical terrain slope increases with the increase of the component of the baseline perpendicular to the radar look direction. A new analytical method, the ratio coherence imagery, is then introduced to highlight total topographic decorrelation against the temporal decorrelation features. The applications of this methodology are demonstrated in selected locations in the Sahara Desert, Algeria, and Almeria, Spain, using ERS-1 and ERS-2 SAR data.
\end{abstract}

Index Terms-Coherence, critical terrain slope, ratio coherence imagery, SAR interferometry, topographic decorrelation.

\section{INTRODUCTION}

$\mathbf{M}$ ULTITEMPORAL interferometric SAR (InSAR) coherence imagery is a useful tool to detect and monitor the randomly changing land surfaces by measuring the degree of temporal decorrelation between the time-separated multiple SAR acquisitions [1]-[3]. This technology has been refined by separating the temporal decorrelation factor from other possible decorrelation sources such as thermal noise, baseline, topography, misregistration, bias on coherence estimation, and atmospheric effects [4]-[12]. Among these decorrelation factors, baseline and topographic factors are often referred to as spatial decorrelation in that they are relevant to the geometric relations of sensor position and target distribution.

In a relief terrain, topographic decorrelation due to the terrain slope is often the dominant decorrelation factor. This type of decorrelation is an intrinsic property of a side-looking and ranging SAR system. It is so overwhelming, particularly on a foreshortened or layover slope, that the coherence drops down dramatically toward zero. The low coherence features on these slopes can easily be misinterpreted in the InSAR coherence imagery as an unstable land surface subject to rapid random change even for a highly stable slope. The total topographic decorrelation, especially in an area where the local terrain slope is equal

Manuscript received December 13, 1999; revised June 5, 2000. The computing and image processing facilities were supported by the University of London Inter-Collegiate Research Services.

The authors are with the T. H. Huxley School of Environment, Earth Sciences, and Engineering, Imperial College of Science, Technology, and Medicine, London SW7 2BP, U.K. (e-mail: hoonyol.lee@ic.ac.uk; j.g.liu@ic.ac.uk).

Publisher Item Identifier S 0196-2892(01)01160-3. or similar to the radar look angle, is generally regarded as an obstacle for SAR interferometry and has not yet been fully investigated. In fact, this particular type of low coherence, once identified, can give us useful information on surface topography.

There have been two different approaches in characterizing spatial decorrelation due to the baseline and the local terrain slope: the geometrical method [4] and the spectral method [6]. In the geometrical method, a baseline decorrelation function is derived by cross-correlating two SAR signals with a given baseline length. This function is simply a Fourier transform (FT) of the radar impulse response. It shows the relationship between the baseline and the coherence level and gives a criterion for choosing the satellite orbits with baselines suitable for InSAR configuration. However, the effect of the local terrain slope, which is the major factor causing the total decorrelation phenomenon on a directly radar-facing slope, has not been fully considered in this method. In the spectral method, the frequency shift of the ground reflectivity spectrum between two SAR observations explains spatial decorrelation caused by both the baseline and the terrain slope. Applying range-varying spectral filtering by using terrain slope information derived from interferogram or pre-existing digital elevation model (DEM), the local coherence can be enhanced at the expense of range resolution. The spectral method has provided a different aspect of understanding spatial decorrelation and has been used to improve the quality of coherence. However, it does not give a quantitative measure of coherence, which is essential for the analysis of the coherence image. Moreover, the spectral method cannot deal with the situation when the shift of the ground reflectivity spectrum exceeds the radar bandwidth, which is the case of the total topographic decorrelation.

In this paper, we present our study on identifying and analyzing the total topographic decorrelation to provide a refined technique for change detection and environmental monitoring using InSAR coherence images. Our study will show the critical conditions for the total topographic decorrelation to occur and how to benefit from this intrinsic limitation on the SAR system by turning it into a useful information for the analysis of coherence image. A modified spatial decorrelation function will be derived geometrically as a function of both the baseline and the local terrain slope. This function will be verified by proving that the geometrical method leads to the same spatial decorrelation function as that from the spectral method. From the modified spatial decorrelation function, a zone of critical terrain slope (or critical incidence angle) can be defined, within which the image of the sloped surface completely loses its coherence under any circumstance. The width of this critical slope angle zone is dependent on the baseline. A new technique, the ratio coherence 
imagery, is then introduced to separate the spatial decorrelation from temporal decorrelation for more effective and reliable interpretation of coherence images. This new technique is then applied to a hyper-arid and relatively flat land surface in the Sahara Desert, Algeria, and a semi-arid and mountainous area in Almeria, Spain, using a series of ERS-1 and ERS-2 SAR data.

\section{THEORY}

\section{A. Spatial Decorrelation Function of Baseline and Topography}

A spatial baseline decorrelation function is given by [4] as

$$
\rho_{\text {spatial }}=1-\frac{2 \cos \theta R_{y} B_{\perp}}{\lambda r}
$$

where

$\theta \quad$ incidence angle;

$\lambda$ radar wavelength;

$r \quad$ slant range;

$B_{\perp} \quad$ baseline component perpendicular to radar look direction;

$R_{y} \quad$ ground range resolution.

Let $\theta_{0}$ represent the nominal incidence angle of the radar on the ellipsoidal Earth (23 for ERS- 1 and ERS-2 at the image center) and $\alpha$ the local terrain slope measured upward from the horizon away from the radar direction so that $\theta=\theta_{0}-\alpha$. The ground range resolution is a function of the local terrain slope as [13]

$$
R_{y}=\frac{c}{2 B_{w}\left|\sin \left(\theta_{0}-\alpha\right)\right|}
$$

where $c$ is the speed of light, and $B_{w}$ is the frequency bandwidth of the transmitted chirp signal. The ground range resolution $R_{y}$ increases rapidly when the surface is nearly orthogonal to the radar beam and becomes infinite if the terrain slope is equal to the nominal incidence angle (i.e., $\alpha=\theta_{0}$ ). Note that $R_{y}$ is practically limited to a finite length because the terrain is definitely not an infinite plane. However, the effect of a large value of $R_{y}$ on the decorrelation is significant in the case of the surface slope facing the radar.

According to (1), the correlation linearly decreases as the ground range resolution increases. This can be understood as the following explanation. SAR achieves resolution by emitting a chirp pulse and then sampling the returned signal with a time sampling interval no longer than the theoretical pulse width after range compression. In each slant range resolution cell of a SAR image, the total scattered field is a coherent summation of radar backscattering from many scatterers within the ground range resolution cell. As the ground range resolution cell increases, the width of the main lobe of the impulse response function broadens and thus, the correlation between two SAR signals diminishes as shown in (1).

Substituting (2) into (1) results in a modified spatial decorrelation function of the baseline and the terrain slope

$$
\rho_{\text {spatial }}=1-A B_{\perp}\left|\cot \left(\theta_{0}-\alpha\right)\right|
$$

where $A=c / \lambda r B_{w}$, a constant for a SAR system. This spatial decorrelation function describes the behavior of topographic

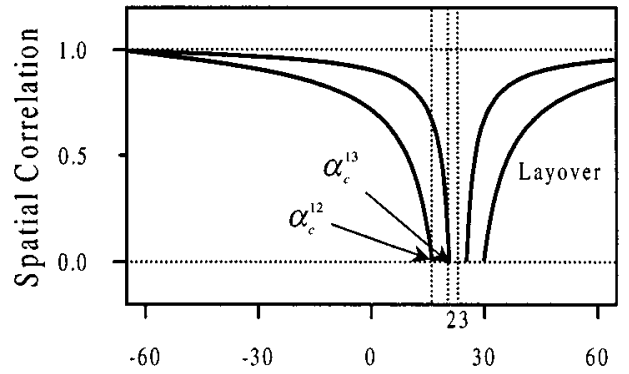

Terrain Slope (degree)

Fig. 1. Spatial decorrelation $\rho_{\text {spatial }}^{12}$ with $300 \mathrm{~m}$ of the baseline perpendicular component $B_{\perp}$ (lower curve) and $\rho_{\text {spatial }}^{13}$ with $100 \mathrm{~m}$ of $B_{\perp}$ (upper curve).

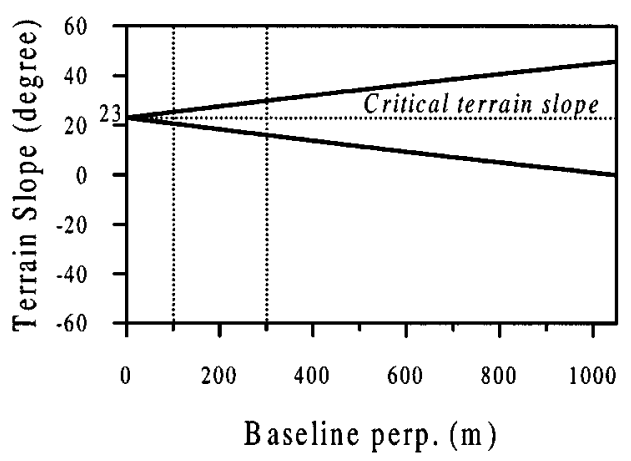

Fig. 2. Critical terrain slopes $\alpha_{c}$ as a function of $B_{\perp}$. Note that the width of the critical terrain slope increases with $B_{\perp}$.

decorrelation as well as baseline decorrelation. For a given baseline, the correlation decreases as the local terrain slope approaches the value of the nominal incidence angle, and the increase of baseline will speed up the deterioration of the correlation (Fig. 1).

The spatial decorrelation function above can be verified by deriving it from a different approach based on the spectral shift in the ground reflectivity spectrum between two radar signals [6],

$$
\Delta f=\frac{c B_{\perp}}{\lambda r} \cot \left(\theta_{0}-\alpha\right) .
$$

Substituting (4) into (3) gives an alternative spatial decorrelation function,

$$
\rho_{\text {spatial }}=1-\left|\frac{\Delta f}{B_{w}}\right| .
$$

If the spectral shift $\Delta f$, a function of the baseline and the local slope, exceeds the bandwidth of a radar chirp signal $B_{w}$, the two SAR spectra become totally disjointed with each other and thus result in total decorrelation. This function is identical to the correlation function derived from spectral misalignment of transfer functions [9], [10]. This means that the two different approaches, the geometrical and the spectral methods, reach the same relationship for the spatial decorrelation function in terms of the baseline and topography.

It should be noted that in derivation of (1)-(5), we assumed the radar impulse response as a square frequency weighting 
function that is a typical radar model. For a different type of impulse response, these equations need to be modified even though the basic relationships remain the same.

\section{B. Critical Terrain Slope (or Critical Incidence Angle)}

From (3), we can define a zone of critical incidence angle $\theta_{c}$ (or critical terrain slope $\alpha_{c}$, where $\alpha_{c}=\theta_{0}-\theta_{c}$ ), within which the spatial decorrelation function falls down to a meaningless value (below zero)

$$
\left|\theta_{c}\right| \leq \tan ^{-1}\left(A B_{\perp}\right)
$$

This critical zone becomes wider as the baseline increases, as shown in Fig. 2. The definition of $A=c / \lambda r B_{w}$ suggests that a SAR system with wider chirp signal bandwidth $B_{w}$ or a longer wavelength $\lambda$ can reduce the critical terrain slope zone and thus the angular range of the total topographic decorrelation. At the image center (midswath), for example, it is $0.4041 \times 10^{-3}$ $\left[\mathrm{m}^{-1}\right]$ for the C-band SAR onboard ERS- 1 and ERS-2, and $0.1174 \times 10^{-3}\left[\mathrm{~m}^{-1}\right]$ for the L-band SAR onboard JERS-1. This simple calculation indicates that an ERS- 1 and ERS-2 InSAR coherence image is about four times more likely to produce the total topographic decorrelation than that of JERS-1.

The total topographic decorrelation is most relevant to the interferometric measurements where the incidence angle equals the actual terrain slope. For example, ERS-1 and ERS-2 SAR has a nominal incidence angle range across the swath of $19.4^{\circ}-26.5^{\circ}$. This angle range coincides with slope angles of moderate relief terrain, a common case of land surface and therefore, the ERS SAR is very likely to produce the total topographic decorrelation. Aircraft measurements on the other hand, may have midswath look angles of $45^{\circ}$ and a range of look angles across the swath of $20^{\circ}-70^{\circ}$. Terrain slope angles equaling the critical incidence angle, in this case, are likely only in a particular range of the image. In practice, the total topographic decorrelation is a problem mainly for interferometric measurements from satellite SAR systems such as SEASAT, ERS-1, ERS-2, JERS-1, and RADARSAT with a relatively narrow incidence angle coinciding with common slope angles of terrain.

\section{Ratio Coherence Imagery: A New Analytical Imagery using Two Coherence Images}

We now introduce a new method for analyzing coherence images. Ignoring other competing decorrelation factors such as thermal noise, misregistration, and atmospheric effects, the total observed correlation of the returned radar signals can be generalized as a product of temporal and spatial correlation as

$$
\rho_{\text {total }}=\rho_{\text {temporal }} \cdot\left(1-A B_{\perp}\left|\cot \left(\theta_{0}-\alpha\right)\right|\right) .
$$

Consider three SAR observations named 1, 2, and 3 in time sequence. A ratio coherence image can then be established by dividing a coherence image with long temporal separation by the other with relatively short temporal separation as

$$
\frac{\rho_{\text {total }}^{13}}{\rho_{\text {total }}^{12}}=\frac{\rho_{\text {temporal }}^{13}}{\rho_{\text {temporal }}^{12}} \cdot \frac{1-A B_{\perp}^{13}\left|\cot \left(\theta_{0}-\alpha\right)\right|}{1-A B_{\perp}^{12}\left|\cot \left(\theta_{0}-\alpha\right)\right|}
$$

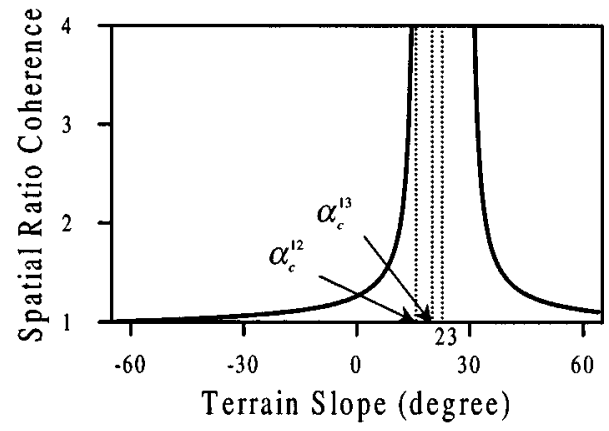

Fig. 3. Spatial ratio coherence $\left(\eta_{\text {spatial }}=\rho_{\text {spatial }}^{13} / \rho_{\text {spatial }}^{12}\right)$. Note the abnormally high value within the zone of critical terrain slope centered at the nominal incidence angle of $23^{\circ}$.

where the superscripts represent the SAR image pair for each coherence image. We can alternatively state that the total ratio coherence consists of a temporal ratio part and a spatial ratio part as

$$
\eta_{\text {total }}=\eta_{\text {temporal }} \cdot \eta_{\text {spatial }} .
$$

Assuming that the temporal change is an accumulated process and as the temporal separations are $\Delta T^{13}>\Delta T^{12}$, the temporal ratio part generally satisfies $\eta_{\text {temporal }} \leq 1$ as $\rho_{\text {temporal }}^{13} \leq$ $\rho_{\text {temporal }}^{12}$. If the baselines are $B_{\perp}^{13} \geq B_{\perp}^{12}$, the total ratio is $\eta_{\text {total }}<1$ for all slopes. For the case of $B_{\perp}^{13}<B_{\perp}^{12}\left(\alpha_{c}^{12}<\alpha_{c}^{13}\right.$ as shown in Figs. 1 and 3, excluding layover zone where $\alpha>\theta_{0}$ for simplicity), however, the spatial ratio part is abnormally high $\left(\eta_{\text {spatial }} \gg 1\right)$ when the terrain slope approaches from zero slope (flat surface) to the critical terrain slope $\alpha_{c}^{12}$, thus producing very high total ratio coherence $\left(\eta_{\text {total }} \gg 1\right)$, as shown in Fig. 3.

Within the zone of $\alpha_{c}^{12}<\alpha<\alpha_{c}^{13}$, the theoretical spatial decorrelation function $\rho_{\text {spatial }}^{12}$ falls down to below zero, but the actual coherence level is limited to $\rho_{\text {spatial }}^{12}=0$, while $\rho_{\text {spatial }}^{13}>0$ (Fig. 1). In this case, the spatial ratio part in (8) may not be well defined theoretically (Fig. 3). However, with constraint to protect division from overflow, the ratio coherence will always result in $\eta_{\text {total }} \gg 1$.

The behavior of the spatial ratio part within the critical terrain slope zone of the shorter baseline $\left(\alpha>\alpha_{c}^{13}\right)$ is ambiguous and cannot be clearly defined because the spatial decorrelation functions of the two coherence images both fall down to below zero. Considering that the estimation of the coherence is an ensemble averaging process with neighboring pixels, the effect of this ambiguity in the theoretical spatial ratio coherence function (8) can be averaged out and become negligible if the critical terrain slope zone of the coherence image with the shorter baseline is very narrow. Otherwise, the ratio coherence will show a random mixture of white and black spots due to the singularity problem of the ratio calculation in the spatial ratio part.

In summary, the feasible working condition for the ratio coherence is as follows:

$$
\eta_{\text {total }}=\frac{\text { Coherence of large } \Delta T \text { and short } B_{\perp}}{\text { Coherence of small } \Delta T \text { and long } B_{\perp}} .
$$

If the baseline of the larger temporal separation coherence image (numerator) is shorter than that of the smaller temporal sepa- 
TABLE I

COHERENCE IMAgES AND THE CRITICAL TERRAIN Slope (SAHARA DESERT, AlgERIA)

\begin{tabular}{l|c|c|c|c}
\hline $\begin{array}{c}\text { Coherence } \\
\text { image }\end{array}$ & $\begin{array}{c}\text { Time } \\
\text { Separation } \\
(\Delta T \text {, days })\end{array}$ & $B_{\downarrow}(\mathrm{m})$ & $\begin{array}{c}\text { Critical incidence } \\
\text { angle }\left(\theta_{\llcorner} \mid \leq\right)\end{array}$ & $\begin{array}{c}\text { Critical terrain slope (with 23 } \\
\text { nominal incidence angle) }\end{array}$ \\
\hline CohI2 & 35 & 263 & $6.0^{\circ}$ & $17.0^{\circ} \sim 29.0^{\circ}$ \\
\hline Coh23 & 350 & 105 & $2.4^{\circ}$ & $20.6^{\circ} \sim 25.4^{\circ}$ \\
\hline Coh 13 & 385 & 368 & $8.4^{\circ}$ & $14.6^{\circ} \sim 31.4^{\circ}$ \\
\hline
\end{tabular}

ration coherence image (denominator), the abnormally bright feature in the ratio coherence image provides an effective identification of the total topographic decorrelation along the radarfacing slopes in contrast to the dark temporal decorrelation features over a stable background in gray.

So far, we have assumed that backscattering from each resolution cell is dominated by scatterers on a tilted plane in an open terrain. In practice, there may be significant contribution from volume scattering effects such as penetration into the dry sand or forest canopy. It is known that the presence of volume scattering reduces coherence level [3]. Moreover, decorrelation from volume scattering also increases as the baseline increases [6], [10]. The phenomenon of the high values of ratio coherence on radar facing slopes will therefore become more significant in the presence of volume scattering. However, these features can only be highlighted over a stable background in gray. If a region is subject to rapid temporal decorrelation and both coherence images in (8) have low values, the ratio coherence image will again be a mixture of randomly distributed black and white spots because of the singularity problem of ratio processing in the temporal ratio part. Therefore, the ratio coherence is only applicable to the area where temporal and volume-scattering decorrelation factors are not significant. This condition is usually satisfied in arid to semi-arid areas where the InSAR coherence technique is to be used to detect temporal changes over a relatively stable background.

It is not always possible to acquire an adequate dataset satisfying the temporal and baseline conditions necessary to establish the ratio coherence imagery in (10). Alternatively, we may use two coherence images, of which the temporal separations do not overlap with each other (for example, $\eta_{\text {total }}=\rho_{\text {total }}^{23} / \rho_{\text {total }}^{12}$, where $\Delta T^{23}>\Delta T^{12}$ ) taking the risk of $\eta_{\text {temporal }}>1$. For an area where surface change processes can be considered as gradually progressive and continuous so that temporal decorrelation is proportional to the temporal separation, the temporal ratio part can then be assumed again to be $\eta_{\text {temporal }}<1$, and the above discussions of ratio coherence imagery will hold for general cases.

However, the assumption between temporal separation and the degree of temporal decorrelation could be overturned by some environmental factors. For example, if an erosion event by an unusual storm or human disturbances have occurred between the first and second image acquisitions only, then the coherence between 1 and $2\left(\rho_{\text {temporal }}^{12}\right)$ would be poor, while the coherence $\rho_{\text {temporal }}^{23}$ might be fine even if $\Delta T^{23}>\Delta T^{12}$. In this case, the nonoverlap temporal separation approach for ratio coherence is not desirable.

The weather conditions can cause more confusion than above. For instance, if there is rainfall at the time of the second image acquisition and dry and stable weather conditions for the first and third images, the coherence between 1 and 2 could be lower than that between 1 and 3. The low coherence between 1 and 2 is largely due to the scattering properties of the microwave that is highly sensitive to the wet surface that permits no penetration. The volume scattering between wet and dry land surface can therefore be considerably different. We, therefore, emphasize that the ratio coherence imagery should be applied in conjunction with accurate precipitation information and weather conditions on the days of SAR image acquisitions. The following applications are the cases of ratio coherence imagery using coherence pairs without time overlapping.

\section{APPLICATIONS}

\section{A. Data Processing for Coherence Estimation}

The coherence is estimated by ensemble averaging the crosscorrelation of SAR complex signals $z_{1}$ and $z_{2}$ within a window of $N$ neighboring pixels:

$$
\rho_{\text {total }}=\frac{\left|\sum_{k=1}^{N} z_{1 k} z_{2 k}^{*} e^{-i \Phi}\right|}{\left[\sum_{k=1}^{N} z_{1 k} z_{1 k}^{*} \sum_{k=1}^{N} z_{2 k} z_{2 k}^{*}\right]^{1 / 2}}
$$

where ${ }^{*}$ is the conjugate of a complex and $e^{-i \Phi}$ a phase factor to compensate for imaging geometry such as earth-flattening and local slope compensation.

A series of signal processing techniques have been applied to produce unbiased estimation of coherence. It includes accurate image co-registration, range spectral filtering, and the use of an optimized sample average window. Two single look complex (SLC) images were co-registered within 1/32 of a pixel that is accurate enough for most InSAR techniques. Range spectral filtering were performed with ellipsoidal Earth approximation to compensate baseline decorrelation. The size of averaging window is determined by a trade-off between unbiased coherence estimation and spatial resolution. Selecting a small averaging window ensures high spatial resolution, but may risk the bias of coherence estimation toward higher values especially in low coherence area [12]. On the contrary, using a large averaging window may underestimate the coherence especially on steep slopes. For our first case study in the Sahara Desert, a relatively small window with 10 pixels only in azimuth direction satisfied both requirements of high contrast coherence image and high spatial resolution in this flat, hyper-arid, and stable region. For the second case study in Almeria, Spain, a window with four pixels in range and 16 pixels in azimuth direction was used.

Range adapting spectral filtering with local slope information, estimated from either the interferogram fringe patterns or pre-existing DEM, can enhance the coherence level in some areas [6]. Furthermore, the removal of the local topographic phase variations can also contribute to an unbiased coherence estimation [12]. However, these methods are not applicable to the total topographic decorrelation areas within the critical terrain slope, because of the unrecoverable noise pattern of phase 


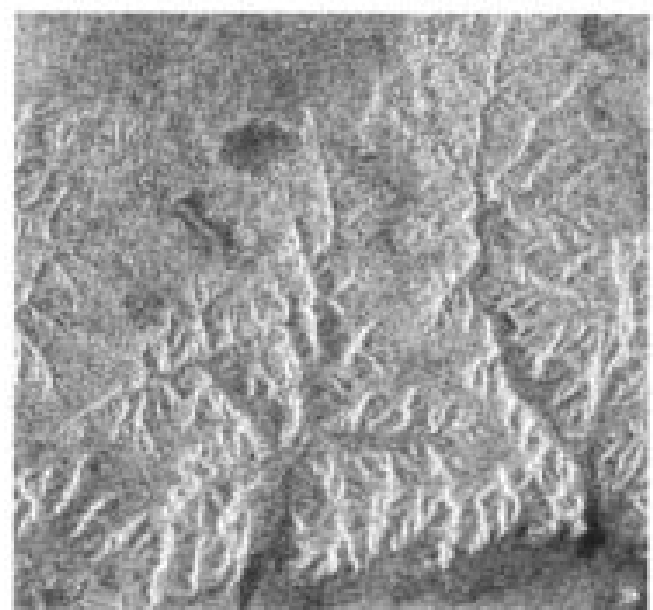

(a)

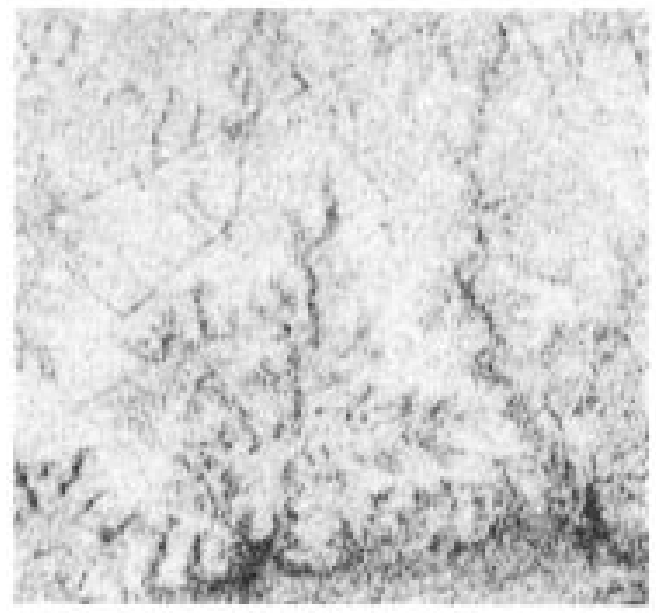

(c)

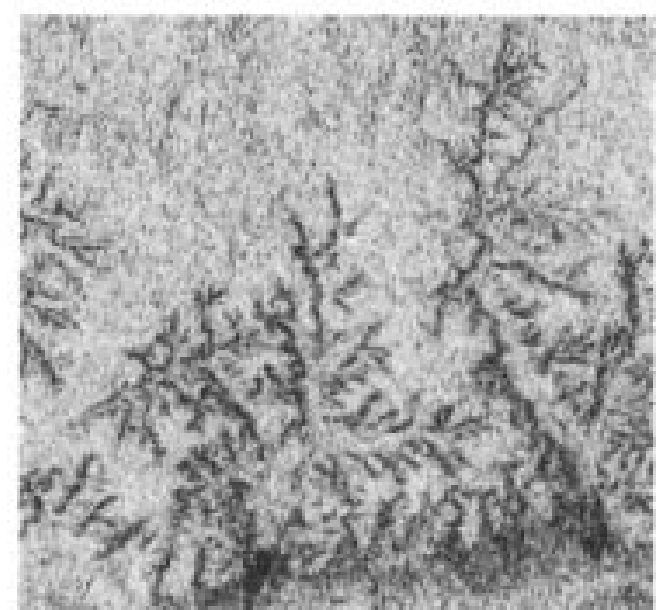

(b)

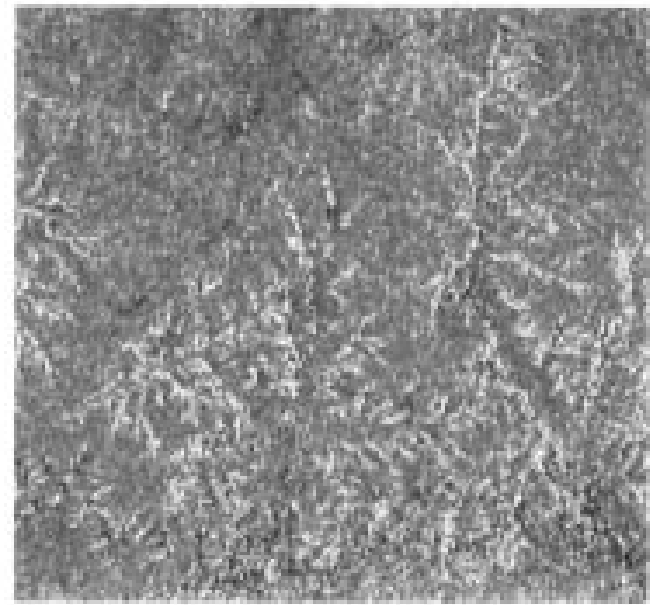

(d)

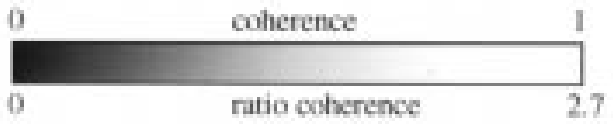

Fig. 4. Topographic decorrelation effect in a gully area $(8 \mathrm{~km} \times 8 \mathrm{~km})$. (a) SAR amplitude image (Alg 3$)$, (b) coherence image Coh 12 with 35 days separation and $263 \mathrm{~m}$ of $B_{\perp}$, (c) coherence image $C o h 23$ with 350 days separation and $105 \mathrm{~m}$ of $B_{\perp}$, and (d) ratio coherence image $C o h 23 / C o h 12$. The low coherence along the gully edges in the coherence images (b) and (c) could be easily mistaken as a temporal decorrelation effect. In the ratio coherence image (d), however, it is clear that the total topographic decorrelation effects along the radar-facing slopes are dominant and highlighted as bright features over a gray background.

in these foreshortened or layover slopes. Obviously, the total topographic decorrelation, in principle, can only be identified and analyzed but cannot be compensated for by these methods.

\section{B. Case Study 1: The Sahara Desert, Algeria-Hyper-Arid, Flat, and Stable Surface}

Three scenes of ERS-1 SAR data of a region in the Sahara desert, Algeria, acquired on September 8, 1992 (Alg1), October 13, 1992 (Alg2), and September 28, 1993 (Alg3) have been processed to generate coherence images. These images were all taken during descending orbits in dry weather conditions [14]. We label a coherence image between $A l g 1$ and $A l g 2$ as $\operatorname{Coh} 12$ (35 days separation and $263 \mathrm{~m}$ of $B_{\perp}$ ) and so on for $\operatorname{Coh} 23$ (350 days separation and $105 \mathrm{~m}$ of $B_{\perp}$ ).
The zones of critical incidence angle of these coherence images are shown in Table I. Among three possible coherence images, Coh 12 and Coh 23 form a ratio coherence image pair that satisfies the condition stated in (10). A ratio coherence image $\operatorname{Coh} 23 / \operatorname{Coh} 12$ is then produced. This ratio coherence configuration is ideal for the discrimination of topographic and temporal decorrelation factors because the temporal separation of $\operatorname{Coh} 23$ is larger than that of $C o h 12$, while the baseline of Coh23 is shorter than that of Coh12. Even though it not a time-overlapping configuration, the temporal ratio can be assumed to be $\eta_{\text {temporal }} \leq 1$ in this hyper-arid and stable region.

An initial study of this area was done by Liu et al. [14] as a case study of the detection and interpretation of random changes over the land surface using multitemporal SAR coherence images. Several interesting coherence features in this 


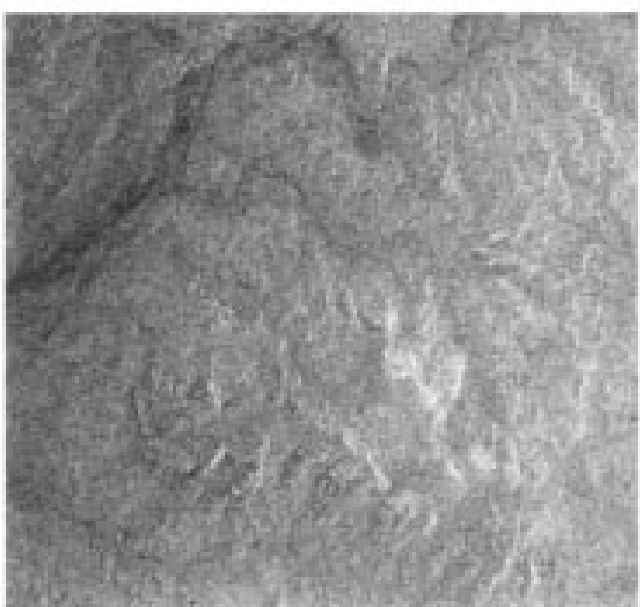

(a)

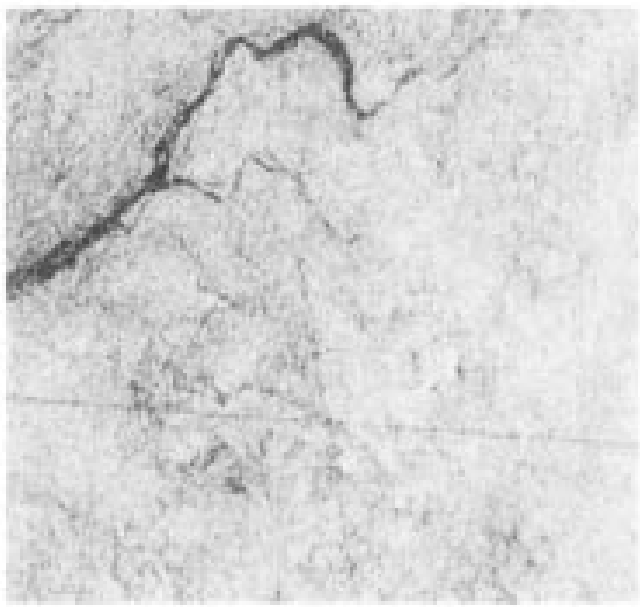

(c)

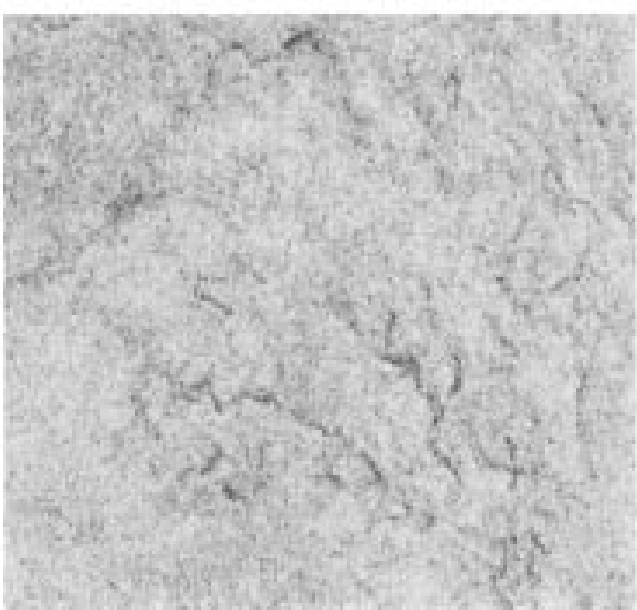

(b)

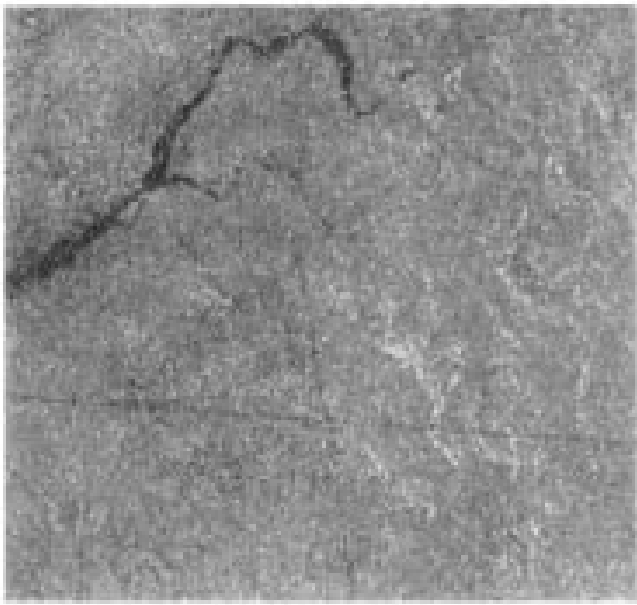

(d)

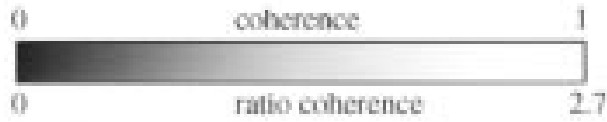

Fig. 5. Example of discriminating the total topographic decorrelation from the temporal decorrelation features (13 km $\times 13 \mathrm{~km})$. (a) SAR amplitude image (Alg3), (b) coherence image Coh 12 with 35 days separation and $263 \mathrm{~m}$ of $B_{\perp}$, (c) coherence image $C o h 23$ with 350 days separation and $105 \mathrm{~m}$ of $B \perp$, and (d) ratio coherence image Coh23/Coh12. Two different decorrelation effects are not distinct from each other in both coherence images (b) and (c), though the gradually losing coherence along major river channels is obvious. In the ratio coherence image (d), these are clearly discriminated as dark river channels of temporal decorrelation (upper left part) and bright radar-facing slopes of the total topographic decorrelation (middle to right) on a stable background in gray.

hyper-arid desert were reported: 1) controversial cases of decoherence features in the gullies showing higher coherence level for larger temporal separation coherence image; 2) ephemeral flood streams and lakes having lost coherence gradually; and 3) low coherence of the sand dunes due to the microscale rapid movement of sand particles by the wind and the volume scattering from radar penetration into the dry sand. In the following section, we will describe how these features appear in the ratio coherence image and will show the advantages of using the ratio coherence imagery.

1) Total Topographic Decorrelation in the Gully Area: Fig. 4 shows the decorrelation features of radar-facing slopes in a dense gully network. Having high coherence on the bottom of the valleys in both Coh 12 and $\operatorname{Coh} 23$ excludes the possibility of rapid fluvial erosion in this hyper-arid re- gion between three SAR observations [14]. Furthermore, the coherence on these radar-facing slopes in $\operatorname{Coh} 23(\Delta T=350$ days) is much higher than that in $\operatorname{Coh} 12$ ( $\Delta T=35$ days), which cannot be explained by their temporal relationship. This is a typical case of the total topographic decorrelation on radar-facing slopes. The theoretical width of the critical terrain slope for $\operatorname{Coh} 23\left(20.6^{\circ} \sim 25.4^{\circ}\right)$ is less than half of that for $\operatorname{Coh} 12\left(17.0^{\circ} \sim 29.0^{\circ}\right)$. Comparing the topographic decorrelation features on Fig. 4(b) and (c), we can conclude that the wider the critical terrain slope range is, the wider the slope area of total decorrelation. On these stable radar-facing slopes, the temporal ratio part can be assumed to be $\eta_{\text {temporal }} \approx 1$, while the spatial ratio part is $\eta_{\text {spatial }} \gg 1$. This results in the highlighted features in the ratio coherence, i.e., $\eta_{\text {total }} \gg 1$. The network of high ratio coherence values along the east-facing 


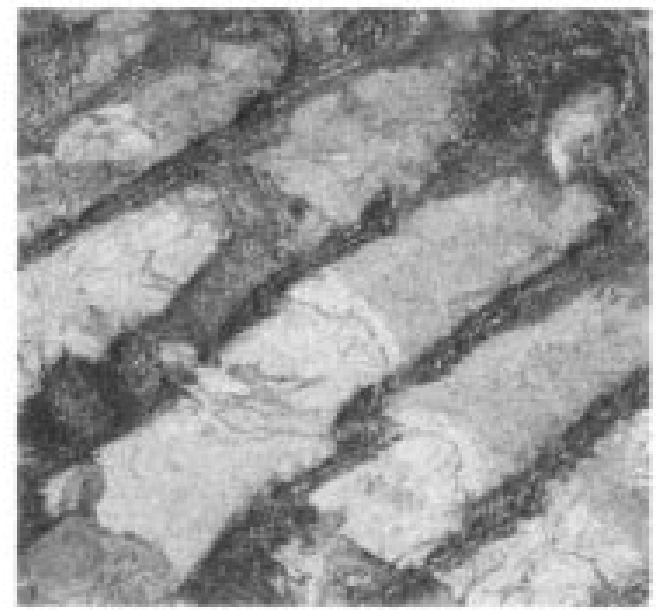

(a)

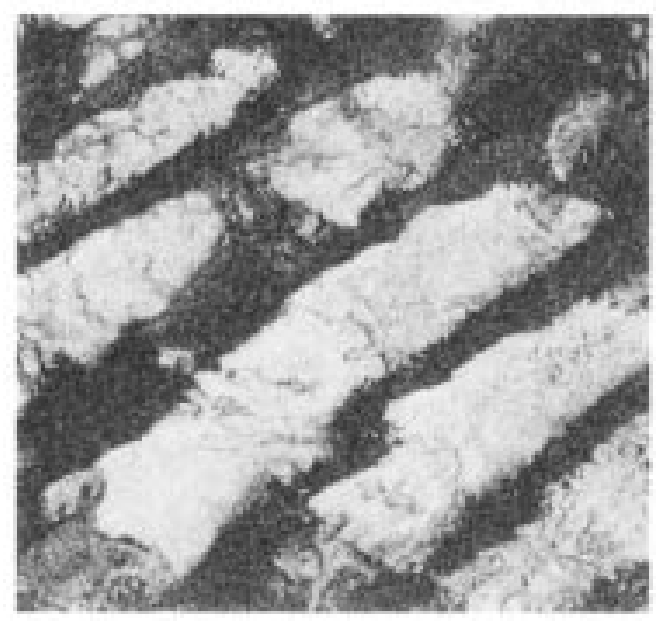

(c)

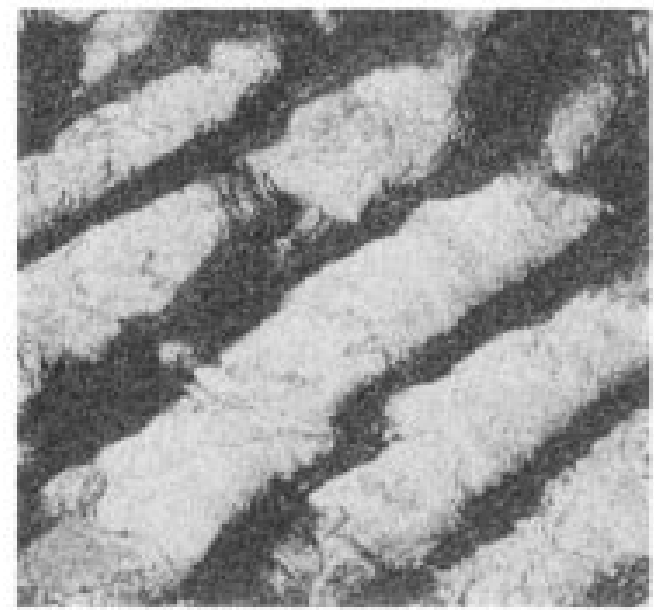

(b)

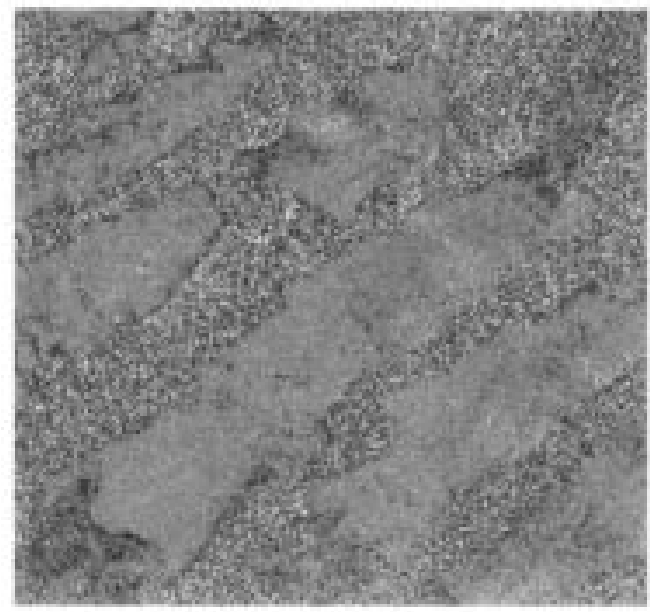

(d)

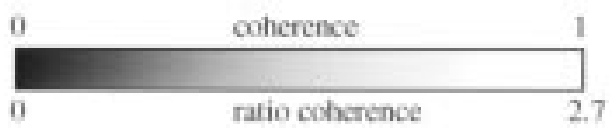

Fig. 6. Rapid temporal decorrelation in the linear type sand dunes due to the continuously moving sand particles and volume scattering (18 km $\times 18 \mathrm{~km}$ ). (a) SAR amplitude image (Alg3), (b) coherence image $C$ oh 12 with 35 days separation and $263 \mathrm{~m}$ of $B_{\perp}$, (c) coherence image $C$ oh 23 with 350 days separation and $105 \mathrm{~m}$ of $B_{\perp}$, and (d) ratio coherence image Coh $23 / \operatorname{Coh} 12$. The low coherence level of the sand dunes in the coherence images (b) and (c) results in random spatial distribution of bright and dark spots over a gray stable background in the ratio coherence image (d).

TABLE II

Ratio Coherence Features (SAHARa Desert, Algeria)

\begin{tabular}{c|c|c|c|c}
\hline Surface type & $\eta_{\text {temporal }}$ & $\eta_{\text {spatial }}$ & $\eta_{\text {total }}$ & $\begin{array}{c}\text { Feature on } \\
\text { ratio coherence image }\end{array}$ \\
\hline Flat bedrock (highly stable) & $\approx 1$ & 1.198 & 1.176 & homogeneously gray \\
\hline $\begin{array}{c}\text { Radar-facing slope (total } \\
\text { topographic decorrelation) }\end{array}$ & $\approx 1$ & $>1$ & $\gg 1$ & bright \\
\hline $\begin{array}{c}\text { Flood stream (gradual temporal } \\
\text { decorrelation) }\end{array}$ & $<1$ & $\approx 1$ & $<1$ & dark \\
\hline $\begin{array}{c}\text { Sand dunes (rapid temporal and } \\
\text { volume scattering decorrelation) }\end{array}$ & random & random & random & $\begin{array}{c}\text { random bright and dark } \\
\text { spots }\end{array}$ \\
\hline
\end{tabular}

sides of the gullies forms eminent bright features over a gray background in (d), isolating the total topographic decorrelation on the radar-facing slopes over the flat and stable bedrock.

2) Discrimination of Topographic and Temporal Decorrelation: Ephemeral Rivers vs. Radar-facing Slopes: Fig. 5 shows
TABLE III

COHERENCE IMAGES AND THE CRITICAL TERRAin Slope (AlMERIA, SPAIN)

\begin{tabular}{l|c|c|c|c}
\hline $\begin{array}{c}\text { Coherence } \\
\text { image }\end{array}$ & $\begin{array}{c}\text { Time } \\
\text { Separation } \\
(\Delta T, \text { days })\end{array}$ & $B_{\perp}(\mathrm{m})$ & $\begin{array}{c}\text { Critical incidence } \\
\text { angle }\left(\left|\theta_{c}\right| \leq\right)\end{array}$ & $\begin{array}{c}\text { Critical terrain slope (with 23 } \\
\text { nominal incidence angle) }\end{array}$ \\
\hline Coh 12 & 70 & 156 & $3.6^{\circ}$ & $19.4^{\circ} \sim 26.6^{\circ}$ \\
\hline Coh 23 & 140 & 20 & $0.5^{\circ}$ & $22.5^{\circ} \sim 23.5^{\circ}$ \\
\hline Coh 13 & 210 & 136 & $3.1^{\circ}$ & $19.9^{\circ} \sim 26.1^{\circ}$ \\
\hline
\end{tabular}

an example of separating the topographic decorrelation from the temporal decorrelation features. The coherence images (b) and (c) show the decorrelation features that cannot be seen in the SAR amplitude image (a). These are the temporal decorrelation features of the erosion process in ephemeral river channels in the upper part of the image, as well as some unknown decorrelation features in the middle to right of the coherence images. These 


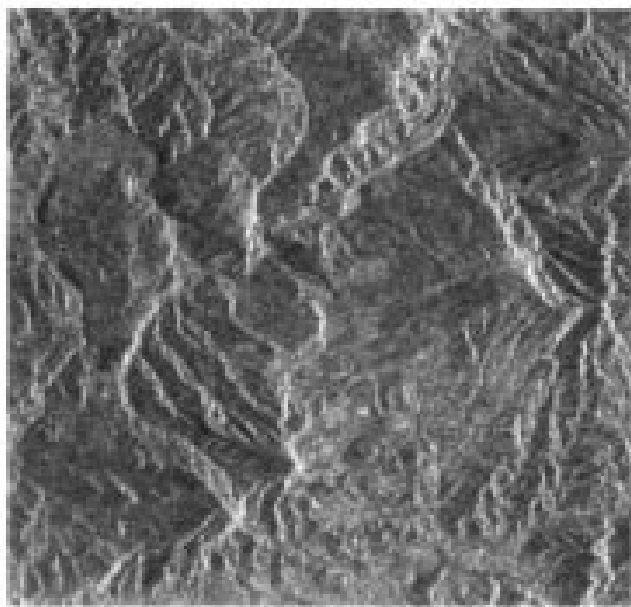

(a)

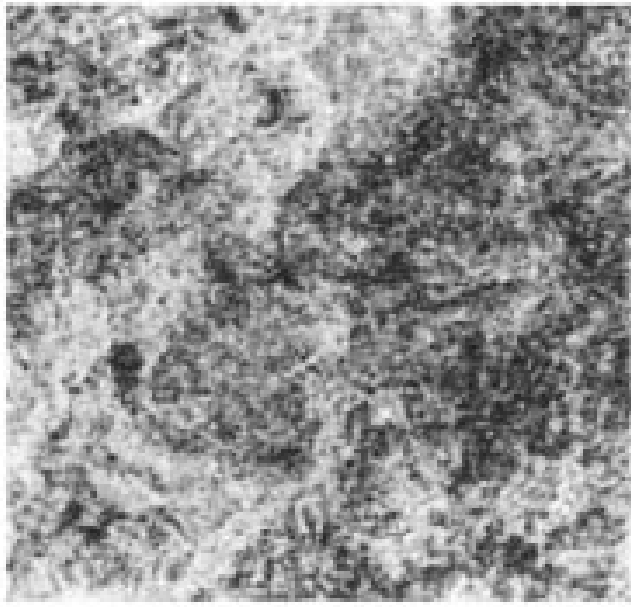

(c)

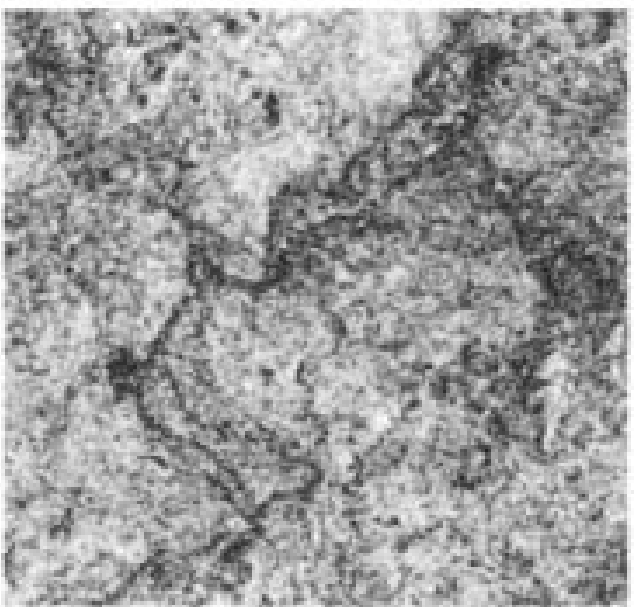

(b)

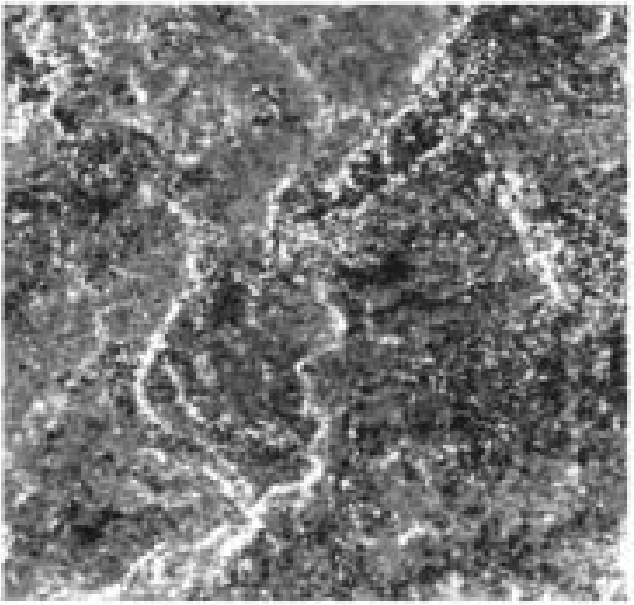

(d)

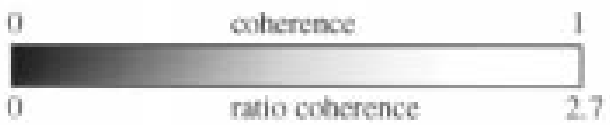

Fig. 7. Ratio coherence imagery in Almeria, Spain $(7.5 \mathrm{~km} \times 7.5 \mathrm{~km})$. (a) SAR amplitude image (Alm 3$)$, (b) coherence image $C o h 12$ with 70 days separation and $156 \mathrm{~m}$ of $B_{\perp}$, (c) coherence image $\operatorname{Coh} 23$ with 140 days separation and $20 \mathrm{~m}$ of $B_{\perp}$, and (d) ratio coherence image Coh23/Coh12. Complex features of decorrelation of different natures in the coherence images (b) and (c) can be clearly discriminated in the ratio coherence image (d). The curved linear features of low coherence highlighted in white are the total topographic decorrelation along the foreshortened slopes. The dark features are of gradual temporal decorrelation from erosion. The black and white noisy spots are from rapid temporal decorrelation of vegetation and agriculture, while gray stands for stable surface.

unknown features, possibly the results of a different decorrelation factor, are not distinguishable from the river channel features in the two coherence images.

Comparing the variation of the coherence features of the river channels in the two coherence images, it is obvious that episodic rainfall and the erosion process had occurred mainly between $A \lg 2$ and $A l g 3$ SAR observations. For these relatively flat river channels, the temporal ratio part is $\eta_{\text {temporal }}<$ 1 , while the spatial ratio part is $\eta_{\text {sptial }} \approx 1$, resulting in a low value of the ratio coherence $\eta_{\text {total }}<1$. On the stable radar-facing slopes near or within the zone of critical terrain slope, the temporal ratio part is $\eta_{\text {temporal }} \approx 1$, while the spatial ratio part is $\eta_{\text {spatial }} \gg 1$, resulting in a high value of the total ratio coherence $\eta_{\text {total }} \gg 1$, as in the case of the gully area. As shown in the ratio coherence image (d), the total topographic decorrelation in the radar-facing slopes is convincingly recognized as bright features against dark features of river channels over a stable and flat background in gray. This example has demonstrated the effectiveness of the ratio coherence imagery for the discrimination between topographic and temporal decorrelation features successfully.

3) Rapid Decorrelation from Temporal Change and Volume Scattering on Sand Dunes: Fig. 6 shows the linear-type sand dunes on stable flat bedrock. Since three SAR scenes were acquired in dry weather conditions, there was a considerable amount of radar penetration into the dry sand, causing volume scattering. The sand dunes show low radar backscattering in the SAR amplitude image (a) due to the volume scattering. 
As shown in the coherence images (b) and (c), the sand dunes also have consistently low coherence level due to both rapid temporal decorrelation and volume scattering. The microscale movement of the sand particles by the wind continuously changes the distribution of scatterers, resulting in rapid temporal decorrelation. The volume scattering also has contributed to decorrelation in the sand dunes [3].

On these sand dunes, the temporal ratio part $\eta_{\text {temporal }}$ can have random spatial distribution of values from extremely high to extremely low because of the singularity behavior of the ratio operation. The slope of each sand dune can also be regarded as continuously varying in microscale, which results in a random variation of the spatial ratio part $\eta_{\text {spatial }}$, thus producing random values of the ratio coherence $\eta_{\text {total }}$. In the ratio coherence image (d), the sand dunes appear as a random mixture of bright and dark spots over gray background of stable and flat bedrock.

Table II summarizes the contribution of the temporal and spatial part of the ratio coherence for the several surface types in the Sahara desert discussed so far.

\section{Case Study 2: Almeria, Spain-A Semi-Arid Area with High Relief Terrain}

We present another application of ratio coherence imagery in a relatively high relief terrain in Almeria, Spain. In this semi-arid area, where annual precipitation is less than 300 $\mathrm{mm}$, badland is a typical landform where the heavily dissected barren terrain is composed of poorly cemented tertiary molasse debris and marls [15]. In this region, surface erosion is an episodic process accompanying occasional rainfall events. However, for a relatively long period (several months to a year), the process can be regarded as cumulative and proportional to the time. We therefore expect erosional phenomena appearing in coherence images as features gradually losing coherence with the increase of temporal separation. Vegetation and human activities (e.g., agriculture) are the two major rapid temporal decorrelation factors. The topographic decorrelation on foreshortened or layover slopes is significant because of this relatively mountainous terrain, as can be seen in the SAR amplitude image (a), which makes correct interpretation of temporal decorrelation features difficult.

Three scenes of ERS-1 and ERS-2 SAR data were used in this case study. The data were obtained on October 3, 1996 (Alm1), December 12, 1996 (Alm2), and May 1, 1997 (Alm3), all in descending orbits. Three possible coherence images are listed in Table III. Among them, the ratio coherence image of Coh $23 /$ Coh 12 meets the condition of (10). Fig. 7 shows two coherence images (b) $\operatorname{Coh} 12$ and (c) $\operatorname{Coh} 23$, and the ratio coherence image (d) Coh $23 / \operatorname{Coh} 12$ of the Rio de Aguas valley nearby the town of Sorbas. Coherence images show complex features of decorrelation from either temporal decorrelation or spatial topographic decorrelation. It is not really possible to distinguish the two using a single coherence image. For instance, the curved linear features of low coherence in Coh 12 image (b) can be easily misinterpreted as temporal decorrelation in streams. With the aid of the ratio coherence image, we can confidently recognize them as the topographic total decorrelation features along the foreshortened or layover slopes of cliff scarps be-
TABLE IV

Ratio Coherence FEATURES (Almeria, Spain)

\begin{tabular}{c|c|c|c|c}
\hline Surface type & $\eta_{\text {temporal }}$ & $\eta_{\text {spatial }}$ & $\eta_{\text {total }}$ & $\begin{array}{c}\text { Feature on } \\
\text { Ratio Coherence Image }\end{array}$ \\
\hline Gypsum plateau (highly stable) & $\approx 1$ & 1.152 & 1.003 & homogeneously gray \\
\hline $\begin{array}{c}\text { Radar facing slope (total } \\
\text { topographic decorrelation) }\end{array}$ & $\approx 1$ & $\gg 1$ & $\gg 1$ & bright \\
\hline $\begin{array}{c}\text { Unconsolidated Tertiary marls } \\
\text { (gradual temporal decorrelation) }\end{array}$ & $<1$ & $\approx 1$ & $<1$ & dark \\
$\begin{array}{c}\text { Vegetation and agriculture (rapid } \\
\text { temporal decorrelation) }\end{array}$ & random & random & random & $\begin{array}{c}\text { random bright and dark } \\
\text { spots }\end{array}$ \\
\hline
\end{tabular}

cause they are highlighted in white in the ratio coherence image (d). The dark areas in (d) are the temporal decorrelation features of progressive erosion in the unconsolidated tertiary molasse debris and marls. The rapid temporal decorrelation of vegetation in the agricultural field in the valley shows randomly distributed black and white spots, while gray in the upper left part of (d) stands for stable gypsum plateau. Table IV summarizes the contribution of the temporal and spatial part of the ratio coherence for the several surface types in this region.

It is interesting to notice that the location of the total topographic decorrelation features partially matches the high backscattering area in the SAR amplitude image (a). Furthermore, comparing two coherence images (b) and (c), Coh 23 is little affected by topographic decorrelation. This is because of the very short baseline of $\operatorname{Coh} 23\left(B_{\perp}=20 \mathrm{~m}\right)$. Nevertheless, we can hardly say that the low coherence features appearing in Coh 12 but missing in $\operatorname{Coh} 23$ are the features of topographic decorrelation only from two coherence images. It is more difficult to pinpoint the features of such kind in practice. Therefore, the ratio coherence image (d) gives a definite identification, and it is much easier to interpret using this than by comparing two coherence images or SAR amplitude images side by side.

\section{CONCLUSIONS}

Topographic decorrelation due to local terrain slope is a dominant decorrelation factor on a surface under direct radar illumination. A highly stable surface may lose its coherence completely when the terrain slope angle is near or within the critical terrain slope zone. This phenomenon can bias the interpretation of a coherence image. The topographic decorrelation has been analyzed by deriving a modified spatial decorrelation function using the geometrical approach. This function was verified by the fact that the same result can also be derived based on the spectral approach. The definition of the critical terrain slope (or critical incidence angle) is then proposed, which, for a given baseline, defines the criterion angle between radar illumination and terrain slope for total decorrelation to occur. It has been shown that the width of the zone of the critical terrain slope (or critical incidence angle) broadens as the baseline increases. As a further development of this study, a new technique, the ratio coherence imagery, is proposed. A ratio between a short baseline, long-temporal separation coherence image (numerator) and a long-baseline, short-temporal separation coherence image (denominator) can effectively enhance and separate topographic decorrelation in white (high ratio values) from temporal decorrelation in black (low ratio values) on a gray background (stable areas). Application examples using ERS-1 and 
ERS-2 SAR data in a hyper-arid flat area in Sahara desert, Algeria, and in a semi-arid area with relief terrain in Almeria, Spain have demonstrated that the technique is useful to eliminate incorrect interpretation of coherence images for detection of random temporal changes on land surface. Weather data, geological information, and adequate temporal and baseline separations between SAR data sets are necessary for correct interpretation of the ratio coherence imagery.

\section{ACKNOWLEDGMENT}

The authors would like to thank NPA Group for providing ERS-1 SAR data of the Sahara Desert, Algeria. ERS-1 and ERS-2 SAR data of Almeria, Spain were provided by the European Space Agency ERS AO3-113 Project. They would also like to thank Prof. H. A. Zebker, Stanford University, Stanford, CA, for his advice on InSAR methodology and software development and PulSAR software by Phoenix Systems and Associates Ltd., U.K., was responsible for SAR and InSAR processing.

\section{REFERENCES}

[1] J. Askne and J. O. Hagberg, "Potential of interferometric SAR for classification of land surfaces," in Proc. Int. Geoscience and Remote Sensing Symp. (IGARSS'93), Tokyo, Japan, 1993, pp. 985-987.

[2] R. Gens and J. L. Van Genderen, "SAR interferometry-Issues, techniques, applications," Int. J. Remote Sens., vol. 17, pp. 1803-1835, Oct. 1996.

[3] J. Hagberg, L. Ulander, and J. Askne, "Repeat-pass SAR interferometry over forested terrain," IEEE Trans. Geosci. Remote Sensing, vol. 33, pp. 331-340, Mar. 1995.

[4] H. A. Zebker and J. Villasenor, "Decorrelation in interferometric radar echoes," IEEE Trans. Geosci. Remote Sensing, vol. 30, pp. 950-959, Sept. 1992.

[5] I. R. Joughin and D. P. Winebrenner, "Effective number of looks for a multilook interferometric phase distribution," in Proc. Int. Geoscience and Remote Sensing Symp. (IGARSS'94), Pasadena, CA, 1994, pp. 2276-2278.

[6] F. Gatelli, A. Monti Guarnieri, F. Parizzi, P. Pasquali, C. Prati, and F. Rocca, "The wavenumber shift in SAR interferometry," IEEE Trans. Geosci. Remote Sensing, vol. 32, pp. 855-865, July 1994.

[7] J. S. Lee, K. W. Hoppel, S. A. Mango, and A. R. Miller, "Intensity and phase statistics of multilook polarimetric and interferometric SAR imagery," IEEE Trans. Geosci. Remote Sensing, vol. 32, pp. 1017-1028, Sept. 1994.

[8] H. Tarayre and D. Massonnet, "Effects of a refractive atmosphere on interferometric processing," in Proc. Int. Geoscience and Remote Sensing Symp. (IGARSS'94), Pasadena, CA, 1994, pp. 717-719.

[9] D. Just and R. Bamler, "Phase statistics of interferograms with applications to synthetic aperture radar," Appl. Opt., vol. 33, no. 20, pp. 4361-4368, 1994

[10] R. Bamler and P. Hartl, "Topical review-Synthetic aperture radar interferometry," Inv. Probl., vol. 14, pp. R1-R54, 1998.

[11] D. Ghiglia, "IFSAR correlation improvement through local slope correction," in Proc. Int. Geoscience and Remote Sensing Symp. (IGARSS'98), Seattle, WA, 1998, pp. 2445-2447.

[12] R. Touzi, A. Lopes, J. Bruniquel, and P. W. Vachon, "Coherence estimation for SAR imagery," IEEE Trans. Geosci. Remote Sensing, vol. 37, pp. 135-149, Jan. 1999.
[13] J. C. Curlander and R. N. McDonough, Synthetic Aperture Radar Systems and Signal Processing: Wiley, 1991.

[14] J. G. Liu, A. Black, H. Lee, H. Hanaizumi, and J. McM. Moore, "Land surface change detection in a desert area in Algeria using multi-temporal ERS SAR coherence images," Int. J. Remote Sens., to be published.

[15] J. G. Liu, H. Lee, and T. Pearson, "Detection of rapid erosion in SE Spain using ERS SAR interferometric coherence imagery," Proc. SPIE, vol. 3868 , pp. 525-535, 1999.

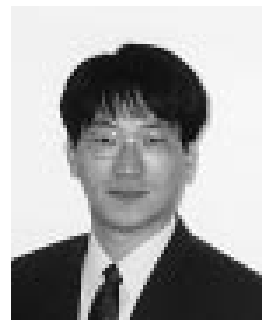

Hoonyol Lee (S'99) was born in Kwangju, Korea, in 1969. He received the B.S. degree in geology in 1995 and the M.S. degree in geophysics in 1997, both from the Department of Geological Sciences, Seoul National University, Seoul, Korea. He is currently pursuing the Ph.D. degree with the Remote Sensing Group, T. H. Huxley School of Environment, Earth Science, and Engineering, Imperial College of Science, Technology, and Medicine, University of London, U.K. He is supported by the Korean Ministry of Education Scholarship and the Overseas Research Student (ORS) Award from the Committee of Vice-Chancellor and Principals (CVCP), U.K.

His research is focused on synthetic aperture radar (SAR), interferometric SAR (InSAR), and differential interferometric SAR (DInSAR) algorithms and software package development and applications to the geomorphology and environmental change. His research interests include SAR focusing, image co-registration, phase unwrapping, image rectification, and surface stability study using coherence and interferogram.

Mr. Lee was awarded the Interactive Session Prize Paper Award at the 1999 IEEE International Geoscience and Remote Sensing Symposium (IGARSS'99, Hamburg, Germany) for his paper, co-authored by Dr. J. G. Liu.

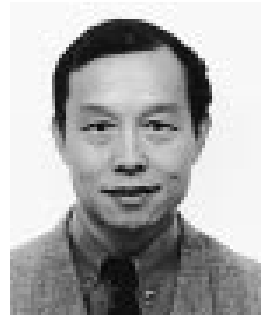

Jian Guo Liu was born in China in 1951. He received the B.Sc. degree in geology from Xi' an Mining Institute, China, in 1978, the M.Sc. degree in remote sensing from the China University of Geosciences, Beijing, in 1982, and the Ph.D. degree in remote sensing and digital image processing from the Imperial College of Science, Technology, and Medicine, London, U.K., in 1991.

He was a Lecturer of remote sensing with the China University of Geosciences from 1982 to 1988 before he went to the U.K. to pursue the Ph.D. degree. He has been with the Imperial College since 1991. He is currently a Research Fellow and a permanent staff member of the T. H. Huxley School of Environment, Earth Science, and Engineering. He developed several novel and popular image processing techniques such as the balance contrast enhancement technique (BCET), the hue RGB composition (HRGB), and the direct decorrelation stretch (DDS). His current research activities are focused on radar interferometry, in particular, the interferometric coherence imagery for random change detection such as sand movement, rapid erosion, and human disturbance (the principal investigator of ESA ERS AO3-113 and ENVISAT AO-803 projects). His teaching activities include undergraduate and M.Sc. image processing courses, M.Sc. advanced data processing techniques for geo-applications and radar remote sensing, and M.Sc. and Ph.D. supervision.

Dr. Liu won the Jerald J. Cook Memorial Award for the best conference paper at the Ninth ERIM Thematic Conferences on Geological Remote Sensing in 1993 and the best session paper award at the Seventh Conference in 1989. 